\title{
Homothetic Preferences Revealed
}

\author{
Jan Heufer and Per Hjertstrand
}




\title{
Homothetic Preferences Revealed*
}

\author{
Jan Heufer ${ }^{\mathrm{a}}$ and Per Hjertstrand ${ }^{\mathrm{b}}$ \\ ${ }^{a}$ Erasmus School of Economics and Tinbergen Institute, Rotterdam \\ ${ }^{\mathrm{b}}$ Research Institute of Industrial Economics, Stockholm
}

November 2017

\begin{abstract}
We propose a method to recover homothetic preferences from choice data with minor optimization or measurement errors. Our method allows for a more detailed graphical analysis to reveal subjects' preferences and to choose appropriate functional forms for parametric analysis. It can also be used to extend applications of the money metric function, such as parametric recoverability as introduced by Halevy et al. (2017). It can also improve nonparametric comparison of preferences as suggested by Heufer (2014).
\end{abstract}

JEL classification: C14; C91; D11; D12.

Keywords: Graphical Analysis; Homotheticity; Money Metric Utility; Recoverability; Revealed Preference.

\section{Introduction}

Under homotheticity, everything there is to know about an agent's preference is implicit in a single indifference set. When there are only two goods, which is common in experimental economics, a preference can therefore be completely summarized graphically by showing a single indifference curve.

A finite set of choice data will, of course, only reveal a part of an agent's preference. This part can be recovered using nonparametric revealed preference techniques as suggested by Varian (1982), who shows how to construct bounds on indifference sets from a set of price and quantity data. Knoblauch (1993) extends this approach by imposing homotheticity, which allows to construct substantially tighter bounds. However, she requires that data are perfectly consistent with homothetic utility maximization, which is very restrictive and almost never the case. Therefore, to the best of the authors' knowledge, the technique has never been applied. In this paper, we show how to implement Knoblauch's method when there are minor violations of homotheticity in the data. Our procedure adjusts the data using the homothetic efficiency approach as suggested by Heufer (2013) and Heufer and Hjertstrand (2017).

Consider Figure 1, which uses choice data from a subject of an experiment conducted by Fisman et al. (2007). The hatched areas show the revealed preferred and revealed worse set for one of the subject's choices using Varian's (1982) technique. These two areas are the bounds on the indifference curve implied by the subject's choices. The indifference curve through the indicated point has to be somewhere in between the hatched parts.

The choices contain some minor violations of homothetic utility maximization, so Knoblauch's (1993) technique cannot be applied directly. However, by adjusting the data, our version of Knoblauch's (1993)

This research did not receive any specific grant from funding agencies in the public, commercial, or not-for-profit sectors. Thanks to Raymond Fisman, Shachar Kariv, and Daniel Markovits for access to their data, and to Linda Hirt-Schierbaum for helpful comments on an earlier draft.

${ }^{* a}$ Erasmus School of Economics and Tinbergen Institute, Erasmus University, 3000 DR Rotterdam, The Netherlands. Email: heufer@ese.eur.nl. ${ }^{b}$ Research Institute of Industrial Economics, Box 5565, SE-102 15, Stockholm, Sweden. Email: per.hjertstrand@ifn.se. 


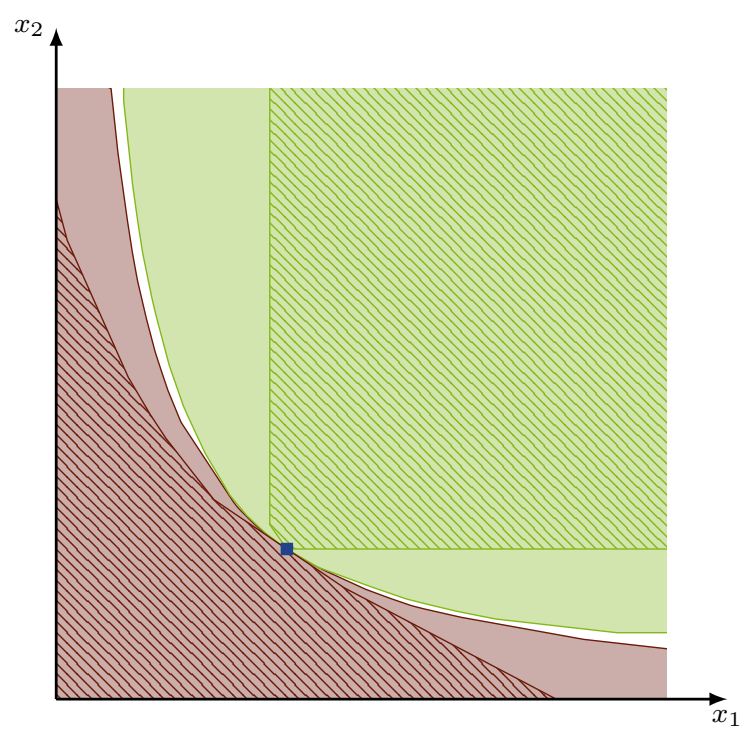

Figure 1: Recovered bounds on preferred and worse sets using data from Fisman et al. (2007): With (solid) and without (hatched) using homothetic recoverability (subject 14).

technique can provide bounds under homotheticity. The solidly shaded areas show the homothetically revealed preferred and worse sets for the same subject. Evidently, the bounds are much tighter, and the sets draw a much clearer picture of the kind of preferences this subject exhibits.

This paper contributes to the possibilities of applying revealed preference methods to choice data, which too often is confined to simply pre-testing data for consistency with utility maximization before conducting further analysis. By showing how to practically recover preferences from choice data under homotheticity, the tighter bounds make applications based on recovered preferences more attractive. As seen in Figure 1, the approach also lends itself to a graphical analysis of the data, which can be used to summarize a subject's preference and use this to decide which functional form to be estimated.

Figure 1 provides a clear illustration of how useful the approach can be. The recovered sets make use of the homothetically revealed preferred relation, which extends the standard revealed preference relation. Every application that makes use of the recovered bounds on revealed preferred and worse sets can benefit from the tighter bounds as long as the assumption of homotheticity can be justified. For example, Halevy et al. (2017) estimate parameters by minimizing a loss function corresponding to the money metric index proposed by Varian (1990). As the bounds on the money metric function can be tightened using homothetic revealed preferences, our approach can be used to estimate models by optimally exploiting the information contained in choice data under homotheticity.

Another application is to improve the revealed preference method to compare preferences of decision makers in terms of their risk aversion as proposed by Heufer (2014). His approach is based on comparing revealed preferred and worse sets; as more information about subjects' preferences under homotheticity can be recovered, the conclusions from the comparisons can be improved.

The crucial part of our contribution is to show how to make Knoblauch's (1993) technique operational by using homothetic efficiency measures to adjust data which violate homotheticity because of minor optimization or measurement error. Of course, this approach suffers from the same drawbacks associated with imposing assumptions of functional forms of utility. However, this drawback is very limited compared to assuming a specific functional form (such as the popular CES function), as we only impose the assumption of homotheticity without any additional structure that comes with a particular utility function.

The rest of the paper is organized as follows. Section 2.1 introduces the notation and some basic 
definitions. Section 2.2 recalls the necessary tools to measure homothetic efficiency, which is required for the adjustment of data. Section 2.3 shows how the approach of Knoblauch (1993) can be made operational using the adjusted data. Section 3 proposes various applications and illustrates the approach using experimental data. Section 4 concludes.

\section{Theory}

\subsection{Preliminaries}

This section briefly summarizes basic definitions and results. A more extensive overview of the relevant concepts can be found in Sections 2 and 3 of Heufer and Hjertstrand (2017).

We use the following notation: For all $\mathbf{x}, \mathbf{y} \in \mathbb{R}^{L}, \mathbf{x} \geqq \mathbf{y}$ if $x_{i} \geq y_{i}$ for all $i=1, \ldots, L ; \mathbf{x} \geq \mathbf{y}$ if $\mathbf{x} \geqq \mathbf{y}$ and $\mathbf{x} \neq \mathbf{y}$; and $\mathbf{x}>\mathbf{y}$ if $x_{i}>y_{i}$ for all $i=1, \ldots, L$. We denote $\mathbb{R}_{+}^{L}=\left\{\mathbf{x} \in \mathbb{R}^{L}: \mathbf{x} \geqq(0, \ldots, 0)\right\}$ and $\mathbb{R}_{++}^{L}=\left\{\mathbf{x} \in \mathbb{R}^{L}: \mathbf{x}>(0, \ldots, 0)\right\}$. The commodity space is $\mathbb{R}_{+}^{L}$, and the price space is $\mathbb{R}_{++}^{L}$, where $L \geq 2$ is the number of different commodities. A budget set is defined as $B^{i}=B\left(\mathbf{p}^{i}\right)=\left\{\mathbf{x} \in \mathbb{R}_{+}^{L}: \mathbf{p}^{i} \mathbf{x}^{i} \leq 1\right\}$, where $\mathbf{p}^{i}=\left(p_{1}^{i}, \ldots, p_{L}^{i}\right)^{\prime} \in \mathbb{R}_{++}^{L}$ is the price vector, and income is normalized to 1 . We observe $N \geq 1$ different budgets and the corresponding demand of a consumer which is assumed to be exhaustive (i.e., $\left.\mathbf{p}^{i} \mathbf{x}^{i}=1\right)$. The entire set of observations on a consumer is denoted $\Omega=\left\{\left(\mathbf{x}^{i}, \mathbf{p}^{i}\right)\right\}_{i=1}^{N}$.

The bundle $\mathbf{x}^{i}$ is directly revealed preferred to a bundle $\mathbf{x}$, written $\mathbf{x}^{i} \mathrm{R}^{0} \mathbf{x}$, if $\mathbf{p}^{i} \mathbf{x}^{i} \geq \mathbf{p}^{i} \mathbf{x}$; it is strictly directly revealed preferred to $\mathbf{x}$, written $\mathbf{x}^{i} \mathrm{P}^{0} \mathbf{x}$, if $\mathbf{p}^{i} \mathbf{x}^{i}>\mathbf{p}^{i} \mathbf{x}$; it is revealed preferred to $\mathbf{x}$ if there exists a sequence $\mathbf{x}^{j}, \ldots, \mathbf{x}^{k}$, such that $\mathbf{x}^{i} \mathrm{R}^{0} \mathbf{x}^{j} \mathrm{R}^{0} \ldots \mathbf{x}^{k} \mathrm{R}^{0} \mathbf{x}$; this is written as $\mathbf{x}^{i} \mathrm{R} \mathbf{x}$, where $\mathrm{R}$ is the transitive closure of $\mathrm{R}^{0}$. The bundle $\mathbf{x}^{i}$ is strictly revealed preferred to $\mathbf{x}$, written $\mathbf{x}^{i} \mathrm{P} \mathbf{x}$, if $\mathbf{x}^{i} \mathrm{Rx}^{j} \mathrm{P}^{0} \mathbf{x}^{k} \mathrm{R} \mathbf{x}$ for some $j, k=1, \ldots, N$. We say that a utility function $u: \mathbb{R}_{+}^{L} \rightarrow \mathbb{R}$ rationalizes a set of observations $\Omega$ if $u\left(\mathbf{x}^{i}\right) \geq u(\mathbf{y})$ whenever $\mathbf{x}^{i} \mathbf{R}^{0} \mathbf{y}$.

A set of observations $\Omega$ satisfies the Generalized Axiom of Revealed Preference (GARP) if for all $i, j=1, \ldots, N$, it holds that $\left[\right.$ not $\left.\mathbf{x}^{i} \mathrm{P}^{0} \mathbf{x}^{j}\right]$ whenever $\mathbf{x}^{j} \mathrm{R} \mathbf{x}^{i}$. Varian (1982) shows that GARP is a necessary and sufficient condition for the existence of a monotonic and concave utility function that rationalizes the data. He also shows how to recover preferences implicit in the data. For some bundle $\mathbf{x}^{0} \in \mathbb{R}_{+}^{L}$ which was not necessarily observed as a choice, the set of prices which support $\mathbf{x}^{0}$ is defined as

$$
S\left(\mathbf{x}^{0}\right)=\left\{p^{0} \in \mathbb{R}_{++}^{L}:\left\{\left(\mathbf{x}^{i}, \mathbf{p}^{i}\right)\right\}_{i=0}^{N} \text { satisfies GARP and } \mathbf{p}^{0} \mathbf{x}^{0}=1\right\} .
$$

The set $S\left(\mathbf{x}^{0}\right)$ is used to provide boundaries on the indifference set. The set of all bundles which are revealed worse than $\mathbf{x}^{0}$ is

$$
R W\left(\mathbf{x}^{0}\right)=\left\{\mathbf{x} \in \mathbb{R}_{+}^{L}: \text { for all } \mathbf{p}^{0} \in S\left(\mathbf{x}^{0}\right), \mathbf{x}^{0} \mathrm{P} \mathbf{x}\right\}
$$

and the set of all bundles which are revealed preferred to $\mathrm{x}^{0}$ is

$$
R P\left(\mathbf{x}^{0}\right)=\left\{\mathbf{x} \in \mathbb{R}_{+}^{L}: \text { for all } \mathbf{p} \in S(\mathbf{x}), \mathbf{x} \mathrm{P}^{0}\right\} .
$$

The convex hull of a set of points $Y=\left\{\mathbf{y}^{i}\right\}_{i=1}^{M}$ is defined as $\left\{\mathbf{x} \in \mathbb{R}_{+}^{L}\right.$ : there exist $\alpha_{i} \in[0,1], \sum_{i=1}^{M} \alpha_{i}=$ 1 such that $\left.\mathbf{x}=\sum_{i=1}^{M} \alpha_{i} \mathbf{y}^{i}\right\}$; the convex monotonic hull $(C M H)$ of a set of points $Y$ is $C M H(Y)=$ convex hull of $\left(\left\{\mathbf{x} \in \mathbb{R}_{+}^{L}: \mathbf{x} \geq \mathbf{y}^{i}\right.\right.$ for some $\left.\left.i=1, \ldots, M\right\}\right)$. Let int $C M H(Y)$ denote the interior of $C M H(Y)$. Varian (1982) and Knoblauch (1992) show that

$$
\operatorname{int} C M H\left(\left\{\mathbf{x}^{i}: \mathbf{x}^{i} \mathrm{R} \mathbf{x}^{0}\right\}\right) \subseteq R P\left(\mathbf{x}^{0}\right) \subseteq C M H\left(\left\{\mathbf{x}^{i}: \mathbf{x}^{i} \mathrm{R} \mathbf{x}^{0}\right\}\right),
$$


which can be used to easily check if $\mathbf{x} \in R P\left(\mathbf{x}^{0}\right)$ and also if $\mathbf{x} \in R W\left(\mathbf{x}^{0}\right)$, because $\mathbf{x} \in R W\left(\mathbf{x}^{0}\right) \Leftrightarrow \mathbf{x}^{0} \in$ $R P(\mathbf{x})$ (see Varian 1982, Fact 3).

In practice, it is often the case that GARP is violated. That requires the use of efficiency measures to adjust the revealed preference relation in order to remove the violation. A particularly efficient way to do so is the Varian Efficiency Vector (VEV) described in Heufer and Hjertstrand (2017) and based on a suggestion by Varian (1993). They define $\mathbf{x}^{i} \mathrm{R}^{0}\left(v_{i}\right) \mathbf{x}$ if $v_{i} \mathbf{p}^{i} \mathbf{x}^{i} \geq \mathbf{p}^{i} \mathbf{x}$, with $\mathrm{R}\left(v_{i}\right)$ being the transitive closure and $\mathrm{P}^{0}\left(v_{i}\right)$ defined analogously. Then $\mathbf{v}=\left(v_{1}, \ldots, v_{N}\right) \in[0,1]^{N}$ is a VEV if it satisfies $\operatorname{GARP}(\mathbf{v})$ (i.e., $\left[\right.$ not $\left.\mathbf{x}^{i} \mathrm{P}^{0}\left(v_{i}\right) \mathbf{x}^{j}\right]$ whenever $\mathbf{x}^{j} \mathrm{R}\left(v_{j}\right) \mathbf{x}^{i}$ ) and there does not exist an $\mathbf{v}^{\prime} \geq \mathbf{v}$ which satisfies $\operatorname{GARP}\left(\mathbf{v}^{\prime}\right)$. The sets $R P$ and $R W$ can then be based on $\mathrm{R}\left(v_{i}\right)$ instead of R. See Heufer and Hjertstrand (2017) for an implementation and justification of this approach.

\subsection{Testing for Homotheticity and Homothetic Efficiency}

This section briefly introduces the concept of measuring homothetic efficiency. For more details, see Heufer and Hjertstrand (2017).

A utility function is homothetic if it is a positive monotonic transformation of a linearly homogeneous utility function; that is, if $u(\mathbf{x})>u(\mathbf{y})$ then $u(\lambda \mathbf{x})>u(\lambda \mathbf{y})$ for all $\lambda>0$. Varian (1983) introduces a homothetic analogue to GARP and shows that it is necessary and sufficient for homothetic utility maximization:

Definition 1 (Varian 1983). A set of observations $\Omega$ satisfies the Homothetic Axiom of Revealed Preference (HARP) if for all distinct choices of indices $i, j, \ldots, \ell$, it holds that $\left(\mathbf{p}^{i} \mathbf{x}^{j}\right)\left(\mathbf{p}^{j} \mathbf{x}^{k}\right) \cdots\left(\mathbf{p}^{\ell} \mathbf{x}^{i}\right) \geq 1$.

Theorem 1 (Varian 1983). There exists a homothetic, monotonic, continuous, and concave utility function which rationalizes the set of observations $\Omega$ if and only if $\Omega$ satisfies HARP.

Because data rarely satisfies HARP, Heufer (2013) and Heufer and Hjertstrand (2017) develop approaches to measure homothetic efficiency. Similar to the Afriat Efficiency Index (Afriat 1972, also known as the Critical Cost Efficiency Index), their Homothetic Efficiency Index (HEI) is a lower bound that can be interpreted as wasted expenditure. A Homothetic Efficiency Vector (HEV) is a more disaggregated measure of efficiency that provides information about wasted expenditure for each observation rather than a lower bound for the entire data set. To define it, we first need the definition of $\operatorname{HARP}(\mathbf{h})$.

Definition 2 (Heufer and Hjertstrand 2017). A set of observations $\Omega$ satisfies HARP(h) for some $\mathbf{h}=\left(h_{1}, \ldots, h_{N}\right) \in(0,1]^{N}$ if for all $i, j=1, \ldots, N$, it holds that

$$
\left(\frac{\mathbf{p}^{i} \mathbf{x}^{j}}{h_{i}}\right)\left(\frac{\mathbf{p}^{j} \mathbf{x}^{k}}{h_{j}}\right) \cdots\left(\frac{\mathbf{p}^{\ell} \mathbf{x}^{i}}{h_{\ell}}\right) \geq 1
$$

An $\mathrm{HEV}$ is a vector $\mathbf{h}$ such that $\operatorname{HARP}(\mathbf{h})$ is satisfied and there does not exist a vector $\mathbf{h}^{\prime} \geq \mathbf{h}$ such that $\operatorname{HARP}\left(\mathbf{h}^{\prime}\right)$ is also satisfied. In our approach to homothetic recoverability in Section 2.3, we only make use of the HEV, but the the approach could also be based on the HEI.

Heufer and Hjertstrand (2017) propose a linear programming procedure to calculate a first-order approximation of the HEV for observed data. This procedure provides a computationally efficient method for large data sets, and Heufer and Hjertstrand (2017) show that the calculated efficiency vector closely approximates the HEV. An implementation to calculate the HEV for a set of data is available in Matlab ${ }^{\circledR}$ as supplementary material to Heufer and Hjertstrand (2017).

Using the HEV for empirical work is well justified, as one can show that if $\operatorname{HARP}(\mathbf{h})$ is satisfied, there exists a homothetic utility function that comes close to representing the data. For that, we say that a 
utility function $u \mathbf{h}$-rationalizes a set of observations $\Omega$ if $u\left(\mathbf{x}^{i}\right) \geq u(\mathbf{y})$ whenever $\mathbf{x}^{i} \mathrm{R}^{0}\left(h_{i}\right) \mathbf{y}$. Theorem 2 shows the relationship between $\operatorname{HARP}(\mathbf{h})$ and $\mathbf{h}$-rationalization.

Theorem 2 (Heufer and Hjertstrand 2017). For any $\mathbf{h}=\left(h_{1}, \ldots, h_{N}\right) \in(0,1]^{N}$, there exists a homothetic, monotonic, continuous, and concave utility function that $\mathbf{h}$-rationalizes the set of observations $\Omega$ if and only if $\Omega$ satisfies $\operatorname{HARP}(\mathbf{h})$.

\subsection{Homothetic Recoverability}

Following Knoblauch (1993), define for a set of observations which satisfies HARP a scalar

$$
t_{i, m}=\min \left\{\left(\mathbf{p}^{i} \mathbf{x}^{j}\right)\left(\mathbf{p}^{j} \mathbf{x}^{k}\right) \cdots\left(\mathbf{p}^{\ell} \mathbf{x}^{m}\right)\right\},
$$

where the minimum is over all finite sequences $i, j, \ldots, \ell$ between 1 and $N$ inclusive, and $t_{i, i}=1$. Note that we can also have $m=0$ for a bundle $\mathbf{x}^{0}$ that was not observed as a choice. We can compute $t_{i, 0}$ as we do not need a price vector $\mathbf{p}^{0}$. We say that $t_{i, m} \mathbf{x}^{i}$ is homothetically revealed preferred to $\mathbf{x}^{m}$, written $t_{i, m} \mathbf{x}^{i} \mathrm{H} \mathbf{x}^{m}$. The scalar $t=t_{i, m}$ is the smallest value such that $t \mathbf{x}^{i} \mathrm{H} \mathbf{x}^{m}$.

Knoblauch (1993) also shows how to recover homothetic preferences implicit in a set of observations which satisfies HARP. Define the set of bundles which are homothetically revealed preferred to $\mathbf{x}^{m}$ as

$$
H R P\left(\mathbf{x}^{m}\right)=\operatorname{int} C M H\left(\mathbf{x}^{m} \cup \bigcup_{i=1}^{N} t_{i, m} \mathbf{x}^{i}\right) .
$$

The set $\operatorname{HRP}\left(\mathrm{x}^{m}\right)$ is very useful indeed, as Theorem 3 below shows that it describes the set of bundles which any rationalizing homothetic utility function must rank higher than $\mathbf{x}^{m}$. Define the set of bundles which are homothetically revealed worse to $\mathrm{x}^{m}$ as

$$
H R W\left(\mathbf{x}^{m}\right)=\left\{\mathbf{x} \in \mathbb{R}_{+}^{L}: \mathbf{x}^{m} \in H R P(\mathbf{x})\right\} .
$$

As $H R P$ can be easily computed as the convex hull of a finite number of points, it is also easy to test for any bundle if $\mathbf{x} \in H R W\left(\mathbf{x}^{m}\right)$.

Theorem 3 (Knoblauch 1993). If $\Omega$ satisfies HARP, then $\mathbf{x} \in H R P\left(\mathbf{x}^{m}\right)$ if and only if every homothetic, monotonic, continuous, and concave utility function which rationalizes $\Omega$ satisfies $u(\mathbf{x})>u\left(\mathbf{x}^{m}\right)$.

These definitions and results rely on the data satisfying HARP. If the data violate HARP, then there exist observations such that $\left(\mathbf{p}^{i} \mathbf{x}^{j}\right)\left(\mathbf{p}^{j} \mathbf{x}^{k}\right) \cdots\left(\mathbf{p}^{\ell} \mathbf{x}^{i}\right)<1$. These can again be multiplied with each other, ad infinitum. Therefore, the scalars in Eq. 6 are not well defined, and the infimum is 0. Alternatively, one could define the scalars over the shortest simple path, that is, by using every observation only once. However, that would be computationally inefficient (to the point of being completely infeasible for larger data sets), and we would not be able to obtain a result that justifies the approach like the one in Theorem 4 below. We therefore propose an approach that relies on adjusting the data by their homothetic efficiency.

Let $\mathbf{h}$ be an HEV of a set of observations $\Omega$. Define

$$
\tilde{t}_{i, m}=\min \left\{\left(\frac{\mathbf{p}^{i} \mathbf{x}^{j}}{h_{i} h_{j}}\right)\left(\frac{\mathbf{p}^{j} \mathbf{x}^{k}}{h_{j} h_{k}}\right) \cdots\left(\frac{\mathbf{p}^{\ell} \mathbf{x}^{m}}{h_{\ell} h_{m}}\right)\right\} .
$$

where, if $m=0$ for a bundle $\mathbf{x}^{0}$, we set $h_{0}=1$. The reason for this definition is that if we adjust the data, we can only confidently state that $\left(\mathbf{p}^{i} \mathbf{x}^{j} / h_{i}\right) \mathbf{x}^{i}$ is homothetically revealed preferred to $h_{j} \mathbf{x}^{j}$ but not necessarily to $\mathbf{x}^{j}$. Note that $\lambda \mathbf{x} \mathrm{H} \mathbf{y}$ implies $\mathbf{x} \mathrm{H} \mathbf{y} / \lambda$, so we have $\left(\mathbf{p}^{i} \mathbf{x}^{j} /\left[h_{i} h_{j}\right]\right) \mathbf{x}^{i} \mathrm{H}^{j}$. 
We say that $\tilde{t}_{i, m} \mathbf{x}^{i}$ is homothetically revealed preferred at efficiency $\mathbf{h}$ to $\mathbf{x}^{m}$, written $\tilde{t}_{i, m} \mathbf{x}^{i} \tilde{\mathrm{H}} \mathbf{x}^{m}$. To motivate this definition and show that it is useful, we first need to define the set of bundles which are homothetically revealed preferred at efficiency vector $\mathbf{h}$ to $\mathbf{x}^{m}$ as

$$
\widetilde{H R P}_{\mathbf{h}}\left(\mathbf{x}^{m}\right)=\operatorname{int} C M H\left(\mathbf{x}^{m} \cup \bigcup_{i=1}^{N} \tilde{t}_{i, m} \mathbf{x}^{i}\right)
$$

and

$$
\widetilde{H R W}_{\mathbf{h}}\left(\mathbf{x}^{m}\right)=\left\{\mathbf{x} \in \mathbb{R}_{+}^{L}: \mathbf{x}^{m} \in \widetilde{H R P}_{\mathbf{h}}(\mathbf{x})\right\}
$$

Theorem 4 below justifies the use of $\widetilde{H R P}_{\mathbf{h}}$ and $\widetilde{H R W}_{\mathbf{h}}$ : It shows that every homothetic utility function which h-rationalizes the data will not contradict our construction of the homothetically revealed preferred and worse sets.

Theorem 4. If $\Omega$ satisfies $H A R P(\mathbf{h})$, then for every homothetic, monotonic, continuous, and concave $u$ which h-rationalizes $\Omega$, it holds that $\widetilde{H R P}_{\mathbf{h}}\left(\mathbf{x}^{m}\right) \subseteq\left\{\mathbf{x} \in \mathbb{R}_{+}^{L}: u(\mathbf{x}) \geq u\left(\mathbf{x}^{m}\right)\right\}$ and $\widetilde{H R W}_{\mathbf{h}}\left(\mathbf{x}^{m}\right) \subseteq$ $\left\{\mathbf{x} \in \mathbb{R}_{+}^{L}: u(\mathbf{x}) \leq u\left(\mathbf{x}^{m}\right)\right\}$.

The proof can be found in the appendix.

\section{Applications}

In this section we illustrate some of the possible applications of our approach using data from Fisman et al. (2007). The data comes from an experimental dictator game in which a subject (the dictator) had to divide money between himself and some other subject (the recipient). The payoffs for the two subjects can be interpreted as two distinct goods. The transfer rate between dictator and recipient varied, such that for every unit given up by the dictator, the recipient could receive more or less than one unit, which implies different prices for the two goods. Thus, subjects chose from budget sets as defined in Section 2.1. Fisman et al. (2007) estimate CES utility functions for their data. As this function is homothetic, applying the assumption of homotheticity without a particular functional form as we do here is still less restrictive.

The data set is well suited for our illustration because the homothetic efficiency of it has already been analysed in Heufer and Hjertstrand (2017) and the recovered preferences are easy to interpret in terms of social preferences. Furthermore, the 76 subjects chose from 50 different budgets, which provides a rich data set. Note that given the large number of observations, there is already a lot to recover about preferences without homotheticity, so we compare our approach with an already quite informative benchmark. For simplicity, we will refer to the non-homothetic benchmarks as the "standard" case.

\subsection{Graphical Illustrations}

Choi et al. (2007b) already provide a short illustrative graphical analysis of revealed preferred and worse sets for data on risk preferences collected by Choi et al. (2007a). We extend this approach using our homothetically revealed preferred and worse sets. Figure 2 shows the recovered standard and homothetically revealed preferred and worse sets of four subjects of Fisman et al. (2007) with different prototypical preferences. As in Figure 1, the hatched areas show the revealed preferred set $R P(\mathbf{x})$ and the revealed worse set $R W(\mathbf{x})$ using the revealed preference relation adjusted by the VEV. The solidly filled areas show the corresponding homothetically revealed preferred and worse sets, $\widetilde{H R P}_{\mathbf{h}}(\mathbf{x})$ and 
$\widetilde{H R W}_{\mathbf{h}}(\mathbf{x})$, respectively. The figures show the preferred and worse sets of actually chosen bundles that are representative of the respective subjects' choices.

The subject in Figure 2.(a) clearly exhibits almost perfectly selfish preferences, that is, $u\left(x_{1}, x_{2}\right)=x_{1}$. Figure 2.(b) shows the preferences of a subject who considers own payoff and payoff for somebody else to be almost perfect substitutes and is therefore very concerned about the total amount, that is, $u\left(x_{1}, x_{2}\right)=x_{1}+x_{2}$. In sharp contrast, the subject in Figure 2.(c) exhibits preferences that come close to maximizing the minimum payout for the dictator and recipient, that is, they come close to maximizing the Rawlsian welfare function $u\left(x_{1}, x_{2}\right)=\min \left\{x_{1}, x_{2}\right\}$ (Rawls 1971). Figure 2.(d) shows preferences of a subjects who comes close to maximizing a Cobb-Douglas function $x_{1}^{\alpha} x_{2}^{1-\alpha}$ with $\alpha$ close to $1 / 2$. This subject therefore comes close to maximizing the Nash product, that is, $u\left(x_{1}, x_{2}\right)=x_{1} x_{2}$ (Nash 1950), which strikes a balance between inequality and the total amount that is paid out.

Note how much narrower the space between the preferred and worse is compared to the standard sets. Three of the subjects shown here make rather extreme choices (always near one of the axes, or always around the $45^{\circ}$ line), and their preferences can already be easily understood by inspecting the raw choice data. However, most subjects fall in between these extremes, and figures like the ones shown here are very helpful to understand their preferences. An implementation of the graphical approach for two-dimensional data sets in Wolfram Mathematica ${ }^{\circledR}$ as well as an interactive tool ${ }^{1}$ for the data of Fisman et al. (2007) can be obtained from the authors.

\subsection{Money Metric and Parametric Recoverability}

Varian (1982) considers the revealed preference implications for Samuelson's (1974) "money metric" cardinalization of utility. The money metric utility of a bundle $\mathbf{x}^{0}$ at prices $\mathbf{p}^{0}$ is the amount a consumer must spend to obtain the utility associated with $\mathbf{x}^{0}$ at prices $\mathbf{p}^{0}$. Varian provides an approximation of the upper bound on the true money metric function; Knoblauch (1992) shows that this bound is already the best possible bound given the observed data. In Knoblauch (1993) she suggests to base the upper bound not on the revealed preferred but the homothetically revealed preferred set. If the assumption of homotheticity can be justified, this can provide a considerably tighter bound on the money metric.

The difference between the standard money metric and the homothetic money metric quantifies the differences of the extents to which preferences can be recovered from the data. Revisiting the idea of the money metric is also very useful because of the recent application of the money metric function to parametric recoverability by Halevy et al. (2017). When their approach is used to recover preferences of a homothetic utility function, imposing homotheticity on the money metric function does not add further restrictions and optimally exploits the information contained in the data. Blackorby and Donaldson (1988) show that money metric utility functions for arbitrary reference prices are generally not concave unless preferences are homothetic, which also adds to the attractiveness of our approach.

Varian (1982) defines the upper bound of the money metric function as $m^{+}\left(\mathbf{p}^{0}, \mathbf{x}^{0}\right)=\inf _{\mathbf{x} \in R P\left(\mathbf{x}^{0}\right)} \mathbf{p}^{0} \mathbf{x}$. Knoblauch (1992) clarifies some issues and shows that the following more practically useful definition is equivalent:

$$
m^{+}\left(\mathbf{p}^{0}, \mathbf{x}^{0}\right)=\min \mathbf{p}^{0} \mathbf{x}^{i} \text { such that } \mathbf{x}^{i} \mathrm{R} \mathbf{x}^{0} .
$$

\footnotetext{
${ }^{1}$ The tool is a computable document; see https://www.wolfram.com/cdf/.
} 


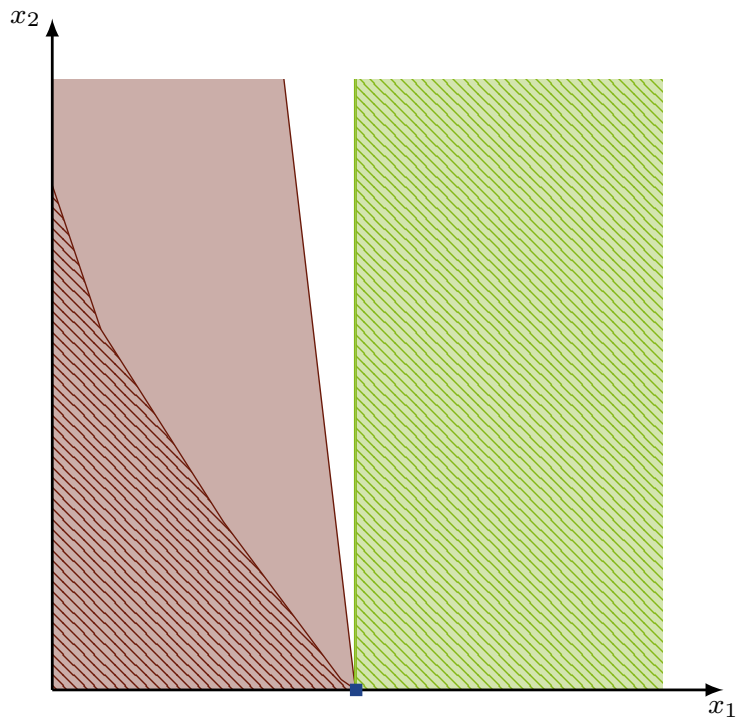

(a)

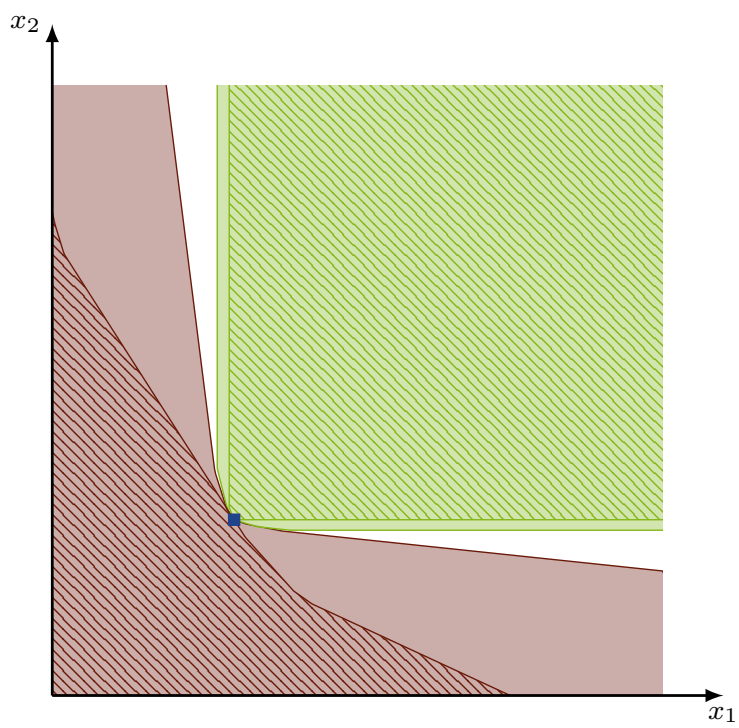

(c)

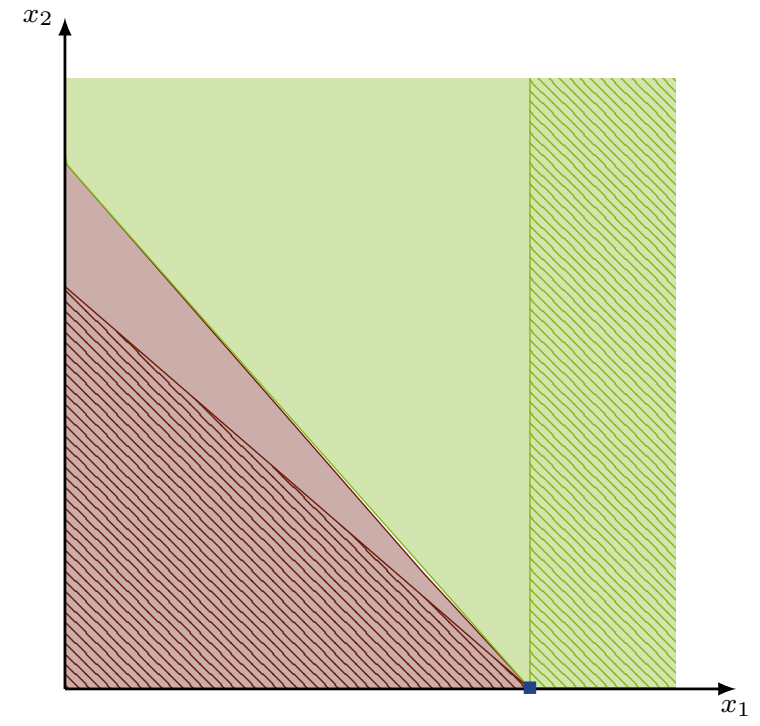

(b)

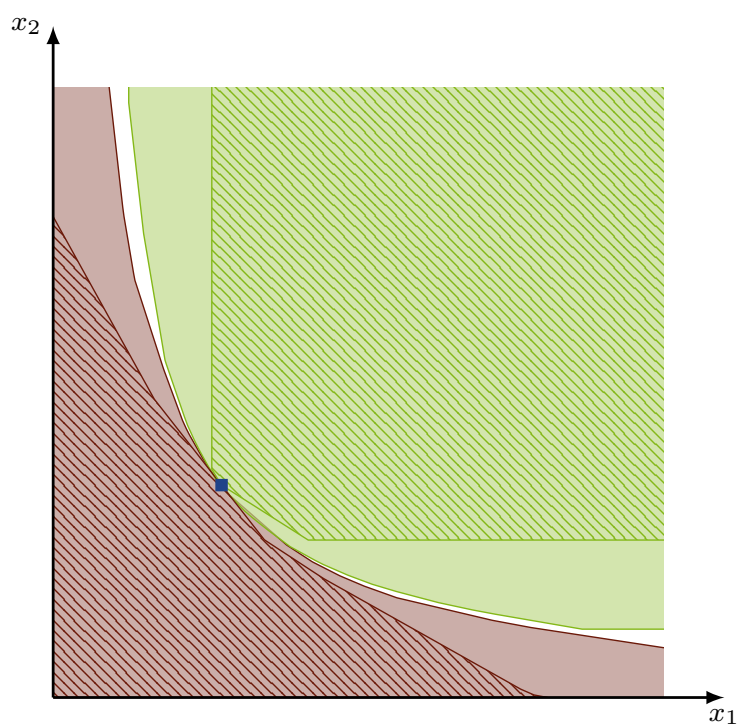

(d)

Figure 2: Standard and homothetically revealed preferred and worse sets for four subjects with prototypical preferences: (a) Selfish preferences, subject 8; (b) perfect substitutes, subject 27; (c) Rawls or Leontief preferences, subject 40; (d) Nash preferences, subject 26. 


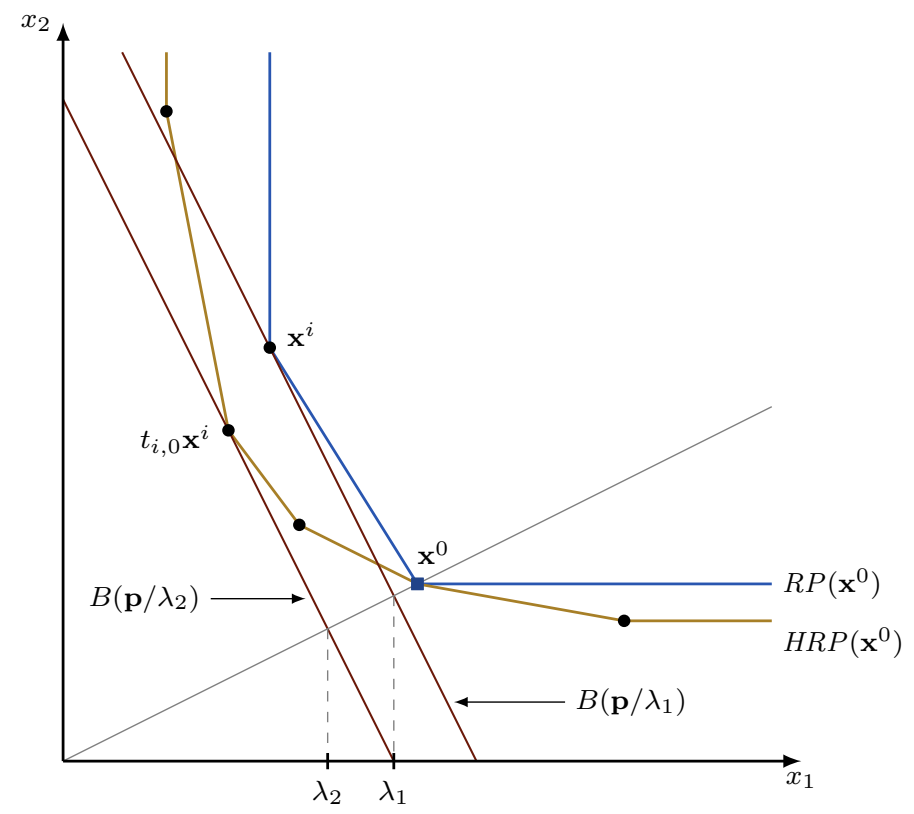

Figure 3: A tighter upper bound for the money metric utility function. The revealed preferred set $R P\left(\mathbf{x}^{0}\right)$ is contained in the homothetically revealed preferred set $H R P\left(\mathbf{x}^{0}\right)$; the figure only shows their boundaries. The upper money metric bound $m^{+}\left(\mathbf{p}, \mathbf{x}^{0}\right)$ is equal to $\lambda_{1}$, as $\mathbf{p x}$ is minimized at $\mathbf{p} \mathbf{x}^{i}$, while the homothetic upper money metric bound $h m^{+}\left(\mathbf{p}, \mathbf{x}^{0}\right)$ is equal to $\lambda_{2}$.

Knoblauch (1993) introduces the homothetic money metric function based on $\operatorname{HRP}\left(\mathbf{x}^{0}\right)$ :

$$
\begin{aligned}
h m^{+}\left(\mathbf{p}^{0}, \mathbf{x}^{0}\right) & =\min _{\mathbf{x} \in H R P\left(\mathbf{x}^{0}\right)} \mathbf{p}^{0} \mathbf{x} \\
& =\min _{i \in\{0, \ldots, N\}}\left\{\mathbf{p}^{0} t_{i, 0} \mathbf{x}^{i}\right\} .
\end{aligned}
$$

Figure 3 illustrates the idea. It shows the bounds of the standard and homothetically revealed preferred sets of an arbitrary bundle $\mathbf{x}^{0}$. The $\mathbf{x}$ that minimizes $\mathbf{p}^{0} \mathbf{x}$ such that $\mathbf{x} \in R P\left(\mathbf{x}^{0}\right)$ is $\mathbf{x}^{i}$; thus, $m^{+}\left(\mathbf{p}^{0}, \mathbf{x}^{0}\right)=\mathbf{p}^{0} \mathbf{x}^{i}=\lambda_{1}$. Then the budget line with $\mathbf{p}^{0}$ divided by $\lambda_{1}$ (or equivalently, the budget line with expenditure $\lambda_{1}$ ) passes through $\mathbf{x}^{i}$. The bound for the homothetic money metric function is $h m^{+}\left(\mathbf{p}^{0}, \mathbf{x}^{0}\right)=\mathbf{p}^{0} t_{i, 0} \mathbf{x}^{i}=\lambda_{2}$, which is a considerably tighter upper bound than $\lambda_{1}$.

We compute the upper bound of the money metric for the data from Fisman et al. (2007). For every subject and every choice $\mathbf{x}^{i}, i \in\{1, \ldots, N\}$, we compute $m^{+}\left(\mathbf{q}^{i}, \mathbf{x}^{i}\right)$ and $h m^{+}\left(\mathbf{q}^{i}, \mathbf{x}^{i}\right)$, where the $\mathbf{q}^{i}$ are normalized price vectors such that $\mathbf{q}^{i} \mathbf{x}^{i}=1$ and $q_{1}^{i}=q_{2}^{i}$. The money metric values therefore reflect how much a dictator would save if the price ratio changes to 1 with full income compensation. We also repeated the analysis with price ratios of $1 / 2$ and 2 and obtained very similar results, which we will not report here.

Figure 4 shows the histogram of the values. It is clear that the homothetic money metric values tend to be lower, indicating that the obtained upper bound is typically tighter. Table 1 shows the means and various percentiles of the two values as well as of their ratios. The mean of all $h m^{+}\left(\mathbf{q}^{i}, \mathbf{x}^{i}\right) / m^{+}\left(\mathbf{q}^{i}, \mathbf{x}^{i}\right)$ is 0.943 , which means that the homothetic bound is about $6 \%$ lower on average. We consider this to be an economically significant improvement on the upper bound of the money metric utility function.

Table 2 shows the mean ratio of the means for all subjects, as well as various percentiles. Note that for the 95 th percentile, the ratios are greater than 1, that is, the homothetic bound is greater than the standard bound. This is because some subjects exhibit very low homothetic efficiency and considerably higher standard efficiency, which requires the homothetically revealed preferred sets to be much more 


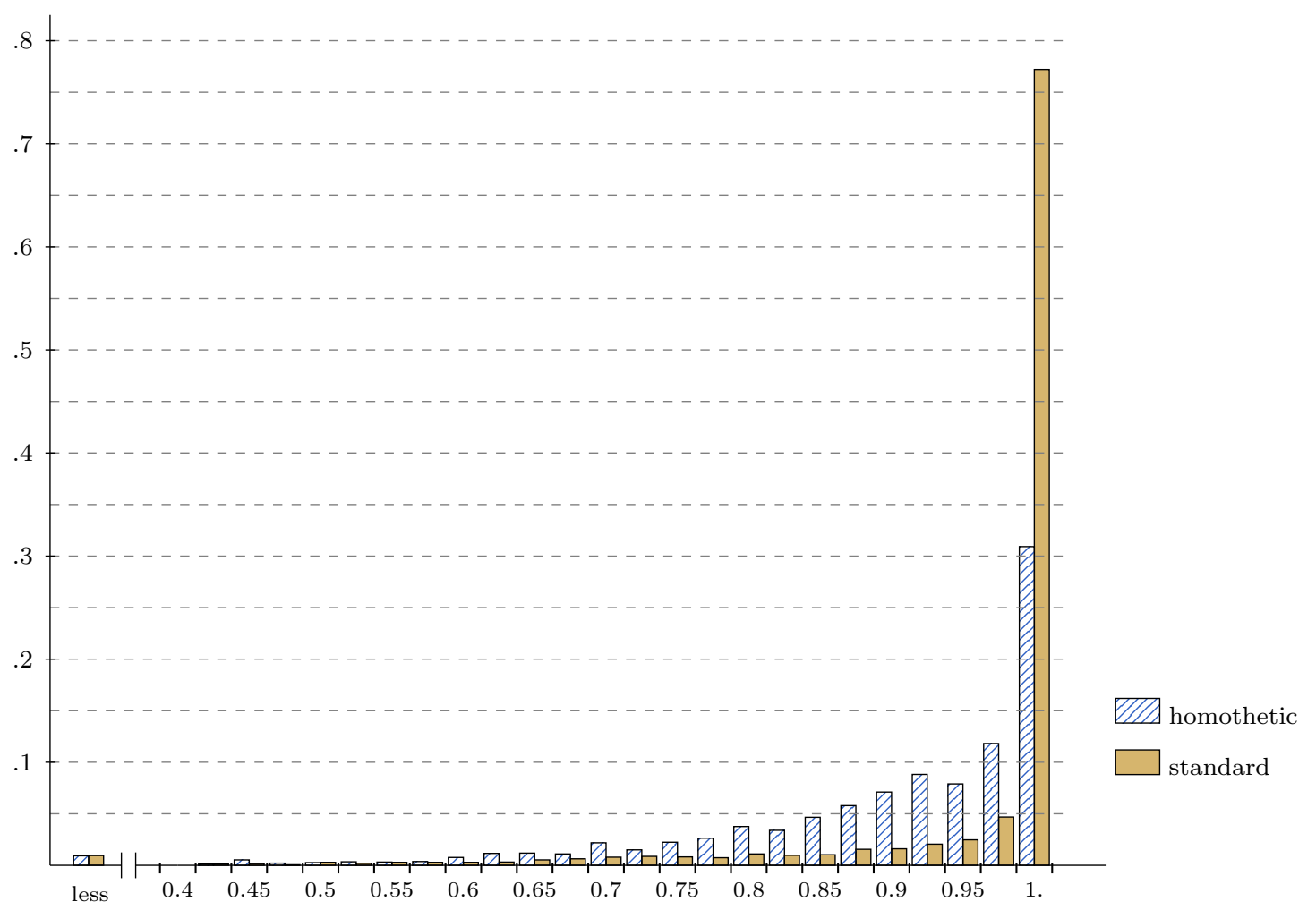

Figure 4: Histogram of the upper bounds of the standard and homothetic money metric utility function for a price ratio of one with normalized price vectors, over all observations of all subjects. Data from Fisman et al. (2007).

\begin{tabular}{|c|c|c|c|c|c|c|}
\hline \multicolumn{7}{|c|}{ Money metric: All observations } \\
\hline & mean & $\mathrm{p} 5$ & $\mathrm{p} 25$ & $\mathrm{p} 50$ & $\mathrm{p} 75$ & p95 \\
\hline$m^{+}\left(\mathbf{q}^{i}, \mathbf{x}^{i}\right)$ (standard) & 0.955 & 0.701 & 0.983 & 1.000 & 1.000 & 1.000 \\
\hline$h m^{+}\left(\mathbf{q}^{i}, \mathbf{x}^{i}\right)$ (homothetic) & 0.887 & 0.624 & 0.839 & 0.928 & 0.982 & 1.000 \\
\hline$\frac{h m^{+}\left(\mathbf{q}^{i}, \mathbf{x}^{i}\right)}{m^{+}\left(\mathbf{q}^{i}, \mathbf{x}^{i}\right)}$ (ratios) & 0.943 & 0.714 & 0.879 & 0.954 & 0.994 & 1.021 \\
\hline
\end{tabular}

Table 1: Upper bounds of the standard and homothetic money metric utility function for a price ratio of one with normalized price vectors, over all observations of all subjects. Data from Fisman et al. (2007).

adjusted than the standard preferred sets. This illustrates that it is important to check if the assumption of homotheticity is justified, as the approach can backfire for preferences which are far from homothetic.

\subsection{Comparability}

Heufer (2014) shows how to use revealed preference relations to operationalize Yaari's (1969) approach to comparative risk aversion. If a risk averse decision maker (DM) $A$ chooses a lottery $\mathbf{x}$ (i.e., a bundle of Arrow-Debreu assets) over a lottery $\mathbf{y}$, even though $\mathbf{y}$ has a higher expected value than $\mathbf{x}$, this implies that the DM did so to avoid the risk involved in $\mathbf{y}$. If another risk averse DM $B$ prefers $\mathbf{y}$ over $\mathbf{x}$, then in this instance, $B$ is clearly less risk averse than $A$. In Heufer (2014), this comparison between two DMs is based on their revealed preferred and worse sets.

The more often we know which of two lotteries a DM prefers, the better we are able to identify differences in risk aversion. Given how much more we can recover using the homothetically revealed preferred and worse sets, it is clear that Heufer's (2014) approach can benefit a lot from basing the comparison on $H R P$ and $H R W$ instead of $R P$ and $R W$ when the assumption of homotheticity is justified. 


\begin{tabular}{|c|c|c|c|c|c|c|c|}
\hline \multicolumn{8}{|c|}{ Money metric ratios } \\
\hline & & \multicolumn{6}{|c|}{ over observations } \\
\hline & & mean & $\mathrm{p} 5$ & p25 & $\mathrm{p} 50$ & p75 & p95 \\
\hline & mean & 0.943 & 0.806 & 0.893 & 0.927 & 0.985 & 1.000 \\
\hline & $\mathrm{p} 5$ & 0.803 & 0.586 & 0.706 & 0.778 & 0.937 & 1.000 \\
\hline over & $\mathrm{p} 25$ & 0.893 & 0.714 & 0.847 & 0.894 & 0.972 & 1.000 \\
\hline \multirow{3}{*}{ subjects } & p50 & 0.943 & 0.812 & 0.901 & 0.940 & 0.990 & 1.000 \\
\hline & p75 & 1.001 & 0.880 & 0.939 & 0.975 & 0.996 & 1.208 \\
\hline & p95 & 1.069 & 0.911 & 0.976 & 0.997 & 1.000 & 1.536 \\
\hline
\end{tabular}

Table 2: Ratios of the bounds of the homothetic and standard money metric function. Data from Fisman et al. (2007).

One can also use the money metric utility function to compare two DMs. When $p_{1} / p_{2}>q_{1} / q_{2}$, then $m^{+}(\mathbf{p}, \mathbf{x})$ will be relatively greater for a DM who values good 2 more than another DM does. For example, a more generous dictator will benefit more from increasing the transfer rate than a more selfish one. This can also be used to quantify the extent of generosity of subjects. As we have seen, the bounds on the money metric utility are typically considerably tighter when imposing homotheticity, which can again improve the comparability. Halevy et al. (2017) use the money metric index to estimate parameters of a utility function; these parameters can also be used to compare DMs. As we have argued above, parametric recoverability can be improved using our homothetic approach, which improves comparisons based on the parameters.

\section{Conclusion}

We believe that, especially for experimental economics, revealed preference methods have much more to offer than only testing data for consistency with a particular decision model. In this paper we provide methods to recover homothetic preferences from choice data and illustrate how this can be used when data does not perfectly satisfy homotheticity but comes close to it.

A related approach developed in a series of papers (Blundell et al. 2003, 2007, 2008, Blundell et al. 2015) relies on using empirical expansion paths in combination with revealed preference theory. Our main objectives differ as we are less interested in making predictions of how consumer welfare is affected by, for example, price and income changes due to the introduction of new policies. Instead, we focus on preferences on the level of the individual decision maker and on differences among decision makers.

Our methods are applicable to each individual subject in experimental data, which makes it suitable to analyse preferences on an individual level and heterogeneity between subjects. This can be done using graphical exploration, computing tighter upper bounds for money metric utility, parametric recoverability, and nonparametric comparability approaches. Apart from being useful for experimental data, the approach can also be applied to household survey panel data, where income and price changes between periods are usually small. This means that on the one hand, budgets rarely intersect, giving low power and little information about preferences without further assumptions, and on the other hand, the assumption of homotheticity is particularly innocuous as we do not deal with large differences in expenditure between periods. Our method does not rely on homogeneity of preferences to estimate income expansions paths and can therefore be used to analyse heterogeneity between households. 


\section{A Appendix}

Proof of Theorem 4. By induction. Suppose $u$ is homothetic, monotonic, continuous, and concave, and h-rationalizes the data. By definition, we can assume without loss of generality that $u$ is homogeneous of degree 1 . Because $u$ is concave, we only need to consider the vertices of the closure of $\widetilde{H R P}_{\mathbf{h}}$, that is, we only need to check if $u\left(\tilde{t}_{i, m} \mathbf{x}^{i}\right)<u\left(\mathbf{x}^{m}\right)$ is possible.

Step 1 By homogeneity of degree $1, u\left(\left[\mathbf{p}^{i} \mathbf{x}^{m} /\left(h_{i} h_{m}\right)\right] \mathbf{x}^{i}\right)=\left[\mathbf{p}^{i} \mathbf{x}^{m} /\left(h_{i} h_{m}\right)\right] u\left(\mathbf{x}^{i}\right)$. Let $\mathbf{y}=\left[h^{i} \mathbf{x}^{m}\right] /\left[\mathbf{p}^{i} \mathbf{x}^{m}\right]$; then $\mathbf{p}^{i} \mathbf{y}=h_{i}$, and therefore $\mathbf{x}^{i} \mathrm{R}^{0}\left(h_{i}\right) \mathbf{y}$. Suppose $u\left(\left[\mathbf{p}^{i} \mathbf{x}^{m} /\left(h_{i} h_{m}\right)\right] \mathbf{x}^{i}\right)<u\left(\mathbf{x}^{m}\right)$. Then $u\left(\mathbf{x}^{i}\right)<$ $\left(h_{i} h_{m} /\left[\mathbf{p}^{i} \mathbf{x}^{m}\right]\right) u\left(\mathbf{x}^{m}\right)$. But with $h_{m} \leq 1,\left(h_{i} h_{m} /\left[\mathbf{p}^{i} \mathbf{x}^{m}\right]\right) u\left(\mathbf{x}^{m}\right) \leq\left(h_{i} /\left[\mathbf{p}^{i} \mathbf{x}^{m}\right]\right) u\left(\mathbf{x}^{m}\right)=u(\mathbf{y})$. Then $u\left(\mathbf{x}^{i}\right)<u(\mathbf{y})$, but $\mathbf{x}^{i} \mathbf{R}^{0}\left(h_{i}\right) \mathbf{y}$, so $u$ cannot $\mathbf{h}$-rationalize $\Omega$. Thus, $\left(\left[\mathbf{p}^{i} \mathbf{x}^{m}\right] / h_{i} h_{m}\right) u\left(\mathbf{x}^{i}\right) \geq u\left(\mathbf{x}^{m}\right)$.

Step 2 Assume without loss of generality that

$$
\tilde{t}_{1, n}=\frac{\mathbf{p}^{1} \mathbf{x}^{2}}{h_{1} h_{2}} \frac{\mathbf{p}^{2} \mathbf{x}^{3}}{h_{2} h_{3}} \ldots \frac{\mathbf{p}^{n-1} \mathbf{x}^{n}}{h_{n-1} h_{n}}
$$

and that $\tilde{t}_{1, m}=\tilde{t}_{1, n}\left[\mathbf{p}^{n} \mathbf{x}^{m}\right] /\left(h_{n} h_{m}\right)$. Suppose $\tilde{t}_{1, n} u\left(\mathbf{x}^{1}\right) \geq u\left(\mathbf{x}^{n}\right)$. Then

$$
\tilde{t}_{1, m} u\left(\mathbf{x}^{1}\right)=\tilde{t}_{1, n} u\left(\mathbf{x}^{1}\right) \frac{\mathbf{p}^{n} \mathbf{x}^{m}}{h_{n} h_{m}} \geq u\left(\mathbf{x}^{n}\right) \frac{\mathbf{p}^{n} \mathbf{x}^{m}}{h_{n} h_{m}} \geq u\left(\mathbf{x}^{m}\right),
$$

where the last inequality follows from Step 1 . Thus, $\tilde{t}_{1, m} u\left(\mathbf{x}^{1}\right) \geq u\left(\mathbf{x}^{m}\right)$. By induction, Steps 1 and 2 show that $u\left(\tilde{t}_{i, m} \mathbf{x}^{i}\right) \geq u\left(\mathbf{x}^{m}\right)$ for all $i$ and all homothetic $u$ which $\mathbf{h}$-rationalize $\Omega$. That concludes the proof for $\widetilde{H R P}$. The second part of the theorem then follows from the definition of $\widetilde{H R W}$.

\section{References}

Afriat, S. N. (1972), "Efficiency Estimation of Production Functions," International Economic Review, $13,568-598$.

Blackorby, C. and Donaldson, D. (1988), "Money metric utility: A harmless normalisation?" Journal of Economic Theory, 46, 120-129.

Blundell, R., Browning, M., Cherchye, L., Crawford, I., De Rock, B., and Vermeulen, F. (2015), "Sharp for SARP: Nonparametric Bounds on Counterfactual Demands," American Economic Journal: Microeconomics, 7, 43-60.

Blundell, R., Browning, M., and Crawford, I. (2003), "Nonparametric Engel Curves and Revealed Preference," Econometrica, 71, 205-240.

- (2007), "Improving Revealed Preference Bounds on Demand Responses," International Economic Review, 48, 1227-1244.

— (2008), "Best Nonparametric Bounds on Demand Responses," Econometrica, 76, 1227-1262.

Choi, S., Fisman, R., Gale, D., and Kariv, S. (2007a), "Consistency and Heterogeneity of Individual Behavior under Uncertainty," American Economic Review, 97, 1921-1938.

- (2007b), "Revealing Preferences Graphically: An Old Method Gets a New Tool Kit," American Economic Review, 97, 153-158. 
Fisman, R., Kariv, S., and Markovits, D. (2007), "Individual Preferences for Giving," American Economic Review, 97, 1858-1876.

Halevy, Y., Persitz, D., and Zrill, L. (2017), "Parametric Recoverability of Preferences," Journal of Political Economy, forthcoming.

Heufer, J. (2013), "Testing Revealed Preferences for Homotheticity with Two-Good Experiments," Experimental Economics, 16, 114-124.

- (2014), "Nonparametric Comparative Revealed Risk Aversion," Journal of Economic Theory, 153, $569-616$.

Heufer, J. and Hjertstrand, P. (2017), "Homothetic Efficiency: Theory and Applications," Journal of Business \& Economic Statistics.

Knoblauch, V. (1992), "A Tight Upper Bound on the Money Metric Utility Function," American Economic Review, 82, 660-663.

— (1993), "Recovering Homothetic Preferences," Economics Letters, 43, 41-45.

Nash, J. F. (1950), "The Bargaining Problem," Econometrica, 18, 155-162.

Rawls, J. (1971), A Theory of Justice, Cambridge: Harvard University Press.

Samuelson, P. A. (1974), "Complementarity: An Essay on the 40th Anniversary of the Hicks-Allen Revolution in Demand Theory," Journal of Economic Literature, 12, 1255-1289.

Varian, H. R. (1982), "The Nonparametric Approach to Demand Analysis," Econometrica, 50, 945-972.

— (1983), "Non-parametric Tests of Consumer Behaviour," Review of Economic Studies, 50, 99-110.

— (1990), "Goodness-of-Fit in Optimizing Models," Journal of Econometrics, 46, 125-140.

— (1993), "Goodness-of-Fit for Revealed Preference Tests," Working Paper, University of Michigan.

Yaari, M. E. (1969), "Some Remarks on Measures of Risk Aversion and on their Uses," Journal of Economic Theory, 1, 315-329. 